\title{
Research on Interactive Space Design of Campus Takeout in Post-epidemic Era
}

\author{
Zihan Wang ${ }^{1, *}$ Weng $\mathrm{Yu}^{1, \mathrm{a}}$ Wanli Zhang ${ }^{1, \mathrm{~b}}$ Jianing Hao ${ }^{1, \mathrm{c}}$ Weizhou Chen ${ }^{1, \mathrm{~d}}$ \\ ${ }^{1}$ Fuzhou University, Xiamen, Fujian 361000, China \\ ${ }^{a}$ Email:1139288952@qq.com \\ ${ }^{b}$ Email:1730985591@qq.com \\ ${ }^{c}$ Email:1536411530@qq.com \\ ${ }^{d}$ Email: 858344410@qq.com \\ *Corresponding author. Email: 19154525@qq.com
}

\begin{abstract}
This article analyzes the relationship between the needs of students ordering takeouts and the placement of takeouts in the post-epidemic era. Based on the preliminary survey results and problems presented in the project, the preliminary concept of the project design is proposed, and a series of analysis of the project's feasibility are made: including the current Chinese and foreign theoretical and practical research results and the relationship between the function and structure of the takeout placement device of each department and each grade and the environment of the takeout placement site. Finally, in combination with the particularity of the college, the environment of the takeout placement point and the takeout placement device are designed to complement local conditions.
\end{abstract}

Keywords: Post-epidemic era, Humanization, Interactive space, Takeout placement.

\section{INTRODUCTION}

\subsection{Research Background}

In the post-epidemic era, college students returned to campus, and the canteen has a large flow of people and the dishes cannot meet the needs of students. College students have successively started the takeout order mode. Most schools stipulate that takeout can only be placed at the school gates. The designers asked nearly a thousand students who often order takeouts. Nearly $90 \%$ said it was difficult to find takeouts on the floor or on the table in front of the school, and more than $70 \%$ said they had experience of losing their takeouts. Secondly, takeouts placed on the side of the road or on the ground can easily cause virus infection. ("Figure 1", "Figure 2", "Figure 3", and "Figure 4") For this purpose, the research follows the principle of "designing for the people" and is based on the takeout crowd. Through this design, it is hoped that this design will improve the efficiency of takeout delivery, optimize the environment of takeout storage sites, and improve the quality of campus life of college students.

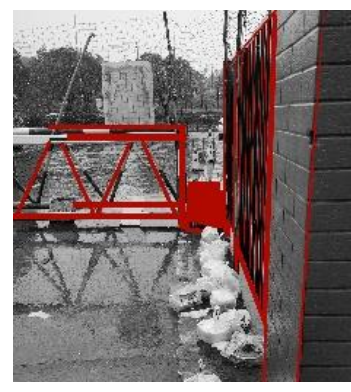

Figure 1 The current situation of the site (source of the picture: on-site shooting)(a).

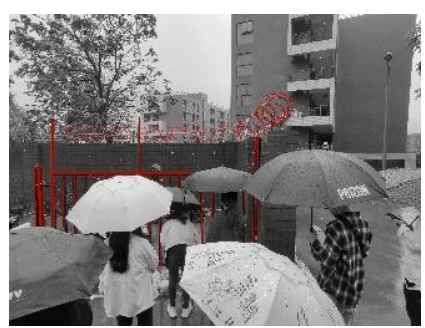

Figure 2 The current situation of the site (source of the picture: on-site shooting) (b). 


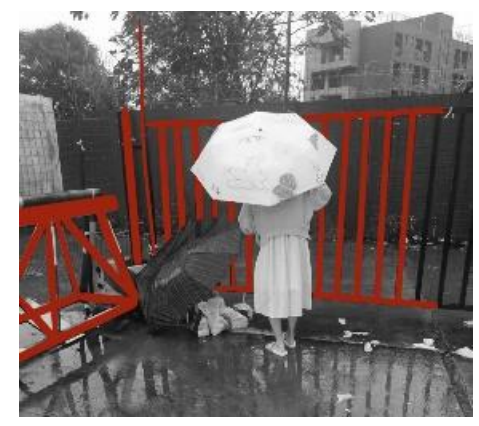

Figure 3 The current situation of the site (source of the picture: on-site shooting) (c).

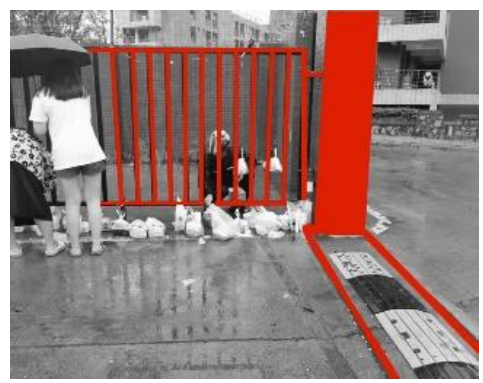

Figure 4 The current situation of the site (source of the picture: on-site shooting) (d).

\subsection{Concept Definition}

\subsubsection{Post-epidemic Era}

At the beginning of 2020, the novel coronavirus pneumonia epidemic has spread all over the world. According to statistics, the number of infections has so far exceeded 180 million. Experts warn that the epidemic will continue to spread across the world, and that mankind should work together to prepare for a protracted battle against the novel coronavirus.

\subsubsection{Humanization}

The core of humanized design lies in "human". The designers put the design focus on human's physical and psychological needs, uphold the principle of "people-oriented", shift the focus of the various functional elements of the research object to the users of the exploration product, and focus on thinking about how to make the design of the product more conducive to the use of the relative population. For example, it is a necessity to conduct sufficient preliminary research and analysis before product development, investigate factors such as the different living habits, consumption concepts and levels, education level and aesthetic tendencies of the population, in order to design high-comfort humanized, convenient, and pleasant scientific products. [1]

\subsubsection{Interaction Design}

"Interaction" refers to the interaction between two or more objects. If the two-way communication interplay is assumed to be an action, people call it "interaction"; if it is assumed to be an object, people call it "interactive interface" or "user interface". Interaction design is a design that makes the interaction feedback between users and products more reasonable, efficient and comfortable. According to the basic principles of interaction design, "The purpose of interaction design is to create products so that users can achieve their goals in the best way." [2]

\section{RESEARCH OVERVIEW IN CHINA AND FOREIGN COUNTRIES}

\subsection{Theoretical Research and Analysis}

\subsubsection{Post-epidemic Solution}

The first is to grasp the experience economy: "Experience economy stage" is a new type of economic life development stage that emerges after grasping the service industry, industrial and commercial economic stages. When material life has been greatly improved and is constrained by the epidemic, people pay more attention to the quality of life, life experience, and health value. [3] The second is to promote technological innovation: artificial intelligence and network economy need to intervene. On the one hand, design should put science and technology into people's lives, and on the other hand, use reasonable ideas to stimulate the development of science and technology, promote the progress of science and technology, and timely integrate scientific and technological achievements into people's lives to create better business models and promotion strategies. The third is to promote demand market in China: it is necessary to promote cultural and tourism integration and urban-rural interaction. Against the background of the epidemic, countries around the world have formed a new development pattern. At this stage, the design has become vigorously pursuing green, innovation, structure, quality, and efficiency. 


\subsubsection{Scientific Techniques and Specific Methods of Humanized Application}

According to research, humanized design usually starts with design psychology, portable design, functional composite design, ergonomics, sensory experience, and other disciplines.

The application methods are:

- Optimizing the model structure: on the basis of the existing functions of the product at this stage, the designers constantly reflect on the uniqueness of the design model, consider the fit of the aesthetic characteristics of the crowd and the crowd's preference for the product, etc., so as to fully consider whether the styling proportion is balanced to make the styling of the product be more in line with the aesthetics of the crowd.

- Designing color: Colors exist depending on the shape of the product, using similar colors, complementary colors and other color matching logic to match, to bring product users the most intuitive and artistic feeling.

- Material selection: The choice of material will affect the affinity between the product itself and the user. Therefore, when choosing the material, it is also necessary to consider whether the material affects the ecological environment, and what kind of material is selected for design can improve the comfort of the product, making the physical and sensory experience of the product user relatively best.

- Functional design: The designers should think about the effectiveness of product functions that can be enhanced in a variety of forms, so that product functions can meet the diverse needs of users. [4]

\subsection{Practical Research Analysis}

In the design of Tsinghua University dormitory furniture, the main design principle is "furniture function extensibility", and the form of bed and desk is adopted to meet more convenient and diverse individual needs. When designing the details of the furniture, it focuses on the design of the basic physiological needs of people:

- Safety - using "stepped" ladders to improve safety.

- Autonomy - providing students with a space based on their own combination.
- Extensibility - The inner side of the locker can be self-punched to adjust the required space scale.

- Convenience - adding a bedside table for easy storage of personal belongings.

Meituan's smart takeout cabinets are designed with convenience as the entry point and humanization as the goal, focusing on simple and efficient access to take-out. Each cabinet can be intelligently insulated and sterilized to keep it clean and hygienic, avoiding secondary infections and reducing the error rate of takeouts.

\section{INTRODUCTION TO THE INTERACTIVE SPACE PROJECT OF POST-EPIDEMIC CAMPUS FOOD DELIVERY}

\subsection{Campus Survey}

From April 28, 2021 to the beginning of May 2021, project team members conduct field inspections and various preliminary phases at the current stage of the takeout placement of the "postepidemic takeout placement site" project and specific issues for the research work. The designers observe and record college students and nearby takeout delivery staff, and conduct interviews and surveys with some students and riders. At the same time, the designers observed nearly a thousand students and nearly a hundred takeout delivery staff in different time periods and different weather conditions, and initially obtained data on the campus takeout logistics model, the number of takeouts and the length of stay under different time points and different weather conditions. ("Figure 5", "Figure 6") 


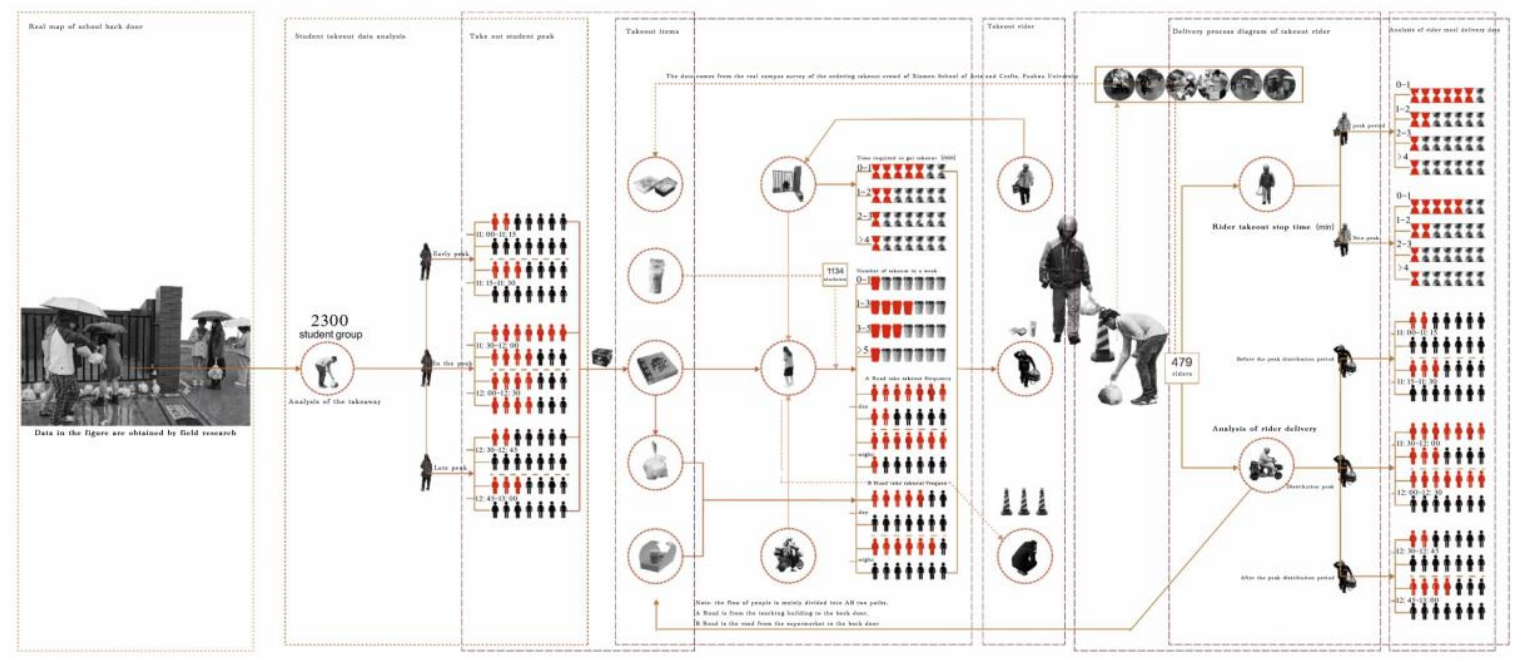

Figure 5 Field survey data analysis diagram of the takeout group (source: drawing by members).
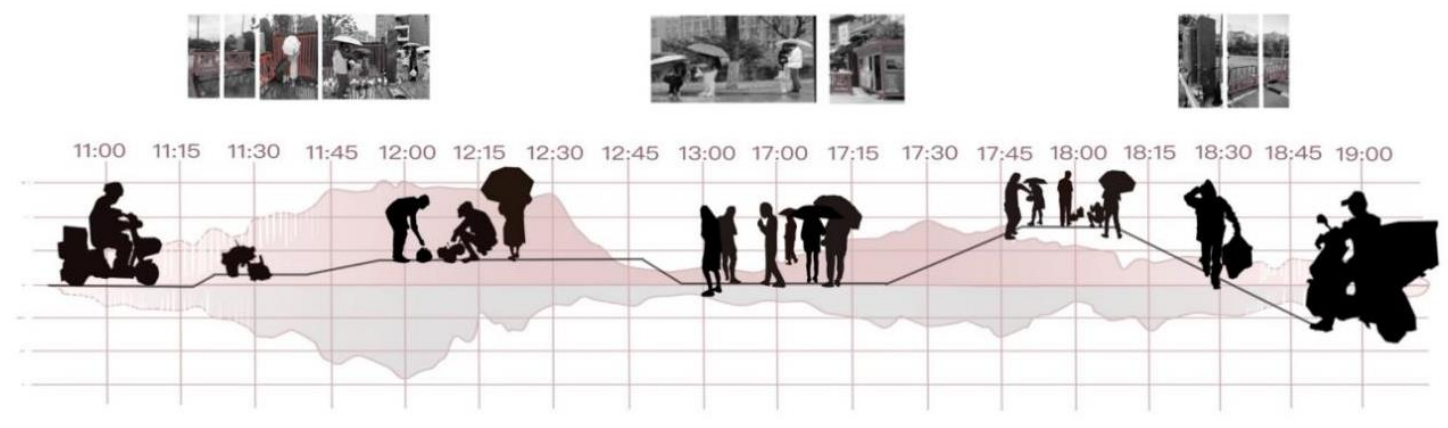

Figure 6 The crest map of taking takeout food at various times on sunny (red) rainy (grey) days (source: drawing by members).

\subsection{Solution Strategy - Combining with Humanization Theory}

Analyzed from the perspective of students, "difficult to find and taking a long time" is a common and serious problem encountered by students ordering takeout groups. In response to this problem, the designers divided the take-out areas for different groups of people, using colors, numbers, etc. The classification method increases the recognition of the takeout storage area of each department and each grade, so that the students who fetch the takeout can see it at a glance, and it is pleasing to the eye. At the same time, in this way, it can add the activity of the takeout storage sites; secondly, "there are no measures for heat preservation, freezing and freshness preservation in the takeout sites, which will easily lead to deterioration of the takeout". Based on these problems, in order to keep the quality and freshness of the takeout, the designers provide thermal insulation and quality assurance devices in the storage area, which fully takes into account the human-oriented concept of "people-oriented" in humanized design, and meets the needs of students who order takeouts; at the same time, there is a phenomenon that "the environment for food delivery is not hygienic, and the order is poor and easy to pick up by mistake". Therefore, the designers adhere to the principle of creating a green and hygienic food delivery environment in the process of designing the food delivery device, and make the design based on ergonomics, sensory experience method, and environmental psychology.

From the perspective of the rider, in response to the status quo that "the size and location of the takeaway is limited when placing the takeout, it is not convenient to place the takeout", the designers organize the placement of the placement device and the size of the internal space according to ergonomics, and guide the takeout rider manual line 
to provide a more convenient delivery method for the riders.

\section{POST-EPIDEMIC CAMPUS TAKEOUT INTERACTIVE SPACE DESIGN PRACTICE}

\subsection{Humanized Design Points of Takeout Placement Devices}

\subsubsection{Taking Ergonomic Principles as the Basis}

The takeout placement device uses human scientific data as samples to make it more in line with students' habits and needs, and can improve the efficiency of fetching takeouts. [5]

\subsubsection{Taking Sensory Experience as the Basis}

The sensory experience of the user in the interactive experience is relatively intuitive. The user's perception of sight, hearing, touch, taste and smell is the basis for designers to research and design. The designers rely on product shape, color and other semantics to convey design information to users, so as to give users a sense of feast. [6]

\subsubsection{Tanking the Functional Composite as the Basis}

The functions based on the needs of psychological behavior are divided into: a. The needs of seeing and being seen - the seats next to the takeout place have a higher utilization rate than other places, because they can provide waiting and rest services for students who fetch takeout, so the demand for seats here is greater. b. The needs for privacy and solitude - in environmental psychology, privacy is currently the psychological need of most people. When designing campus landscape facilities, it is particularly important to choose a suitable location and deal with the surrounding environment. It is necessary to pursue relative privacy while maintaining the overall harmony and unity of the space, so as to provide students with a more private leisure space.

The design principles based on facilities to space are divided into:

- a. Natural environment adaptability principles - To combine the school's natural elements and adapt to local conditions.

- $\quad$ b. The principle of sustainable use of space - To use green and environmentally friendly recyclable materials.

\subsection{Takeout Placement Device Design}

\subsubsection{Description of Device Function Structure}

From a functional point of view, the device provides storage space for takeout and partitions the space inside and outside the school, while achieving the purpose of diverting the crowd and ensuring the hygiene of takeout food to prevent secondary infection. On this basis, heat preservation devices are provided on the left and right for students to heat and cool for the takeouts.

Based on efficient and healthy humanized design, the designers choose aluminum-plastic panels for assembly and construction. According to the ergonomics related theories and the research on the relationship of various take-out scales ("Figure $7 ")$, the designers set the height range of the installation to be $160-170 \mathrm{~cm}$, and the internal left compartment is divided into 3 spaces, each with a size of $120 * 20 * 40 \mathrm{~cm}$, which is used as a freshman to junior takeout storage area; The right compartment is divided into 2 spaces, each with a size of $30 * 40 * 30 \mathrm{~cm}$, which is used as a storage area for seniors, and the total length is set at $150 \mathrm{~cm}$. A $150 * 40 * 70 \mathrm{~cm}$ takeout storage area is set up below the device, and a $40 * 40 \mathrm{~cm}$ partition is used to limit the loss of items. According to the relationship between the scale of the back door structure of the school and the site, in order to maximize the utilization of the site area and rationally guide the movement of the takeout group, a "convex" structure arrangement device is adopted. A cooling and heating platform is set in the junction area of the two devices near the inner side of the school. 


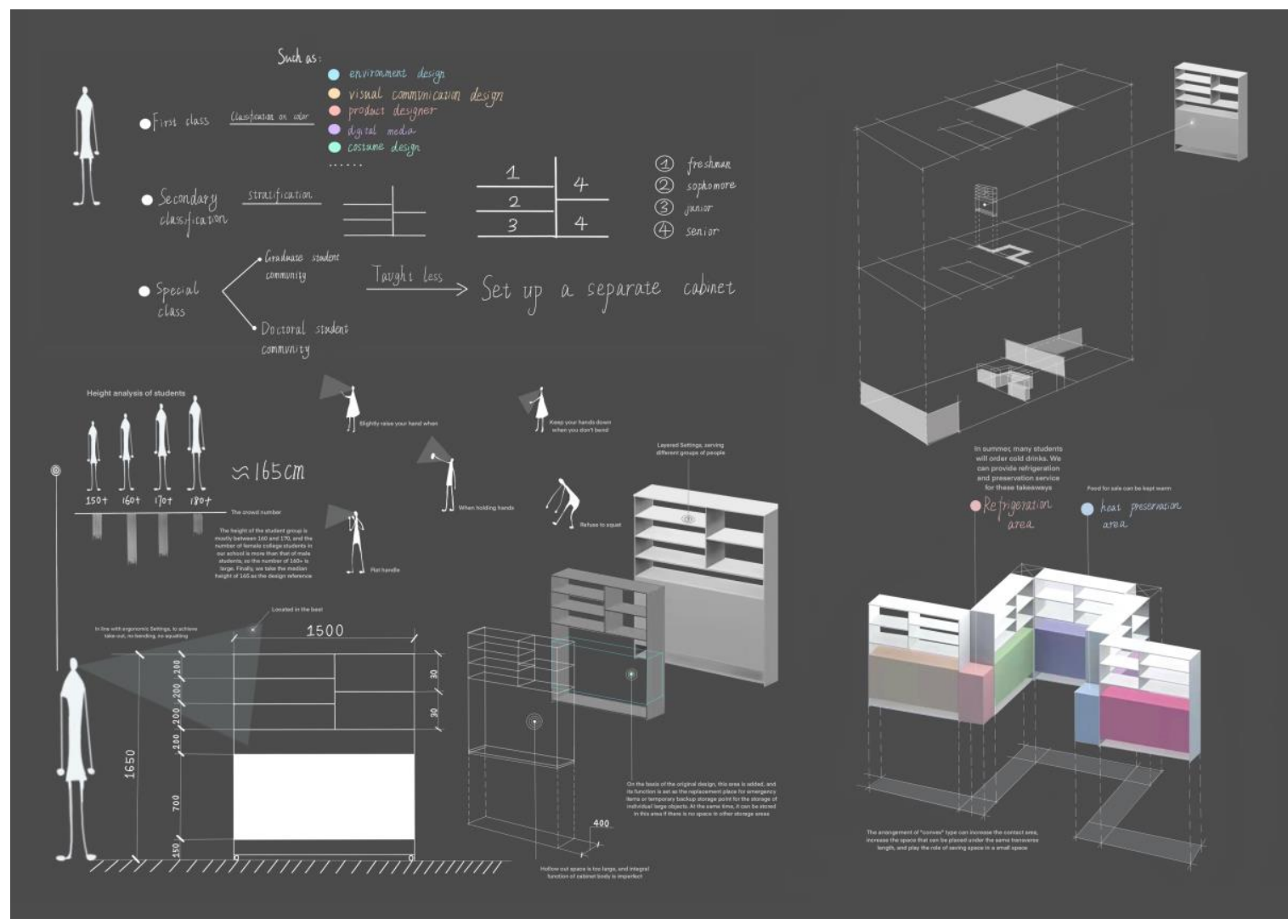

Figure 7 Analysis of the device structure (source: drawing by members).

\subsubsection{Art Form of the Device}

The designers design different shapes, colors and accessories according to different departments to divide the take-out placement devices. For example, the environmental design department can use green as the main color, and use the natural environment as a source of inspiration for pattern design and apply it to the appearance of the device. They can also select elements such as stones, dead branches, and poinciana trees as accessories based on local materials, and distinguishing each grade with digital art on this basis; Another example is to choose white as the main color of the sculpture department, and place some small plaster artworks to echo it; mud yellow and blue and white represent the ceramics department, animation and games represent the number and media department, and the four treasures of the study represent the traditional Chinese painting department... The designers strive to provide clearer visual guidance and psychological hints to students who fetch takeouts, improve the recognition of takeout placement points, and show the characteristics of each department.

\subsection{Description of the Environment Where the Takeout Placement Device Is Located}

\subsubsection{Placement Environment for Takeout}

The designers' school is affiliated to the Jimei District of Xiamen City and is located in the southeast coast of China. It has a subtropical monsoon climate. The average temperature in summer is high and heavy rain often falls. The average temperature in winter is about 15 degrees Celsius with little rainfall. Therefore, on top of the basic functions of the takeaway placement device, the designers use calcium silicate boards to increase the sun and rain protection, and use UV lamps to prevent mosquito interference. At the same time, the designers designed corresponding ground elements as guide signs according to the different visual forms of each department's device to improve the recognition of each department's takeout placement device. In terms of night lighting design, on the basis of lighting, the designers use different visual patterns from various departments to guide people to increase the artistry of the 
takeout storage area and improve the efficiency of students fetching takeout at night.

\subsubsection{Rest Space Environment}

After analyzing the results of the questionnaire, according to the needs of some students who fetch the takeouts, the designers designed leisure spaces around the takeout storage area, divided into public and private spaces, scientifically planned vegetation landscape, artistic treatment of paving styles, innovative design the shape of table and chair, and reasonable set the seat height to provide a comfortable waiting environment, dining environment, and entertainment environment for students who order takeouts.

\section{CONCLUSION}

In the post-epidemic era, we redesigned the campus take-out storage area, based on objective conditions and the needs of the take-out crowd, and conformed to the principles of scientific and humanized design. It is a must for the designers to jointly create and maintain an orderly, stable and efficient social environment, and seek a harmony between science and convenience, efficiency and flexibility from the crowd of ordering takeouts, the crowd of food delivery to the crowd of fetching takeouts and with the attitude of "the people's worries are what I think; the people's thinking is what I do".

\section{AUTHORS' CONTRIBUTIONS}

Zihan Wang contributed to preliminary material collection. Weng Yu has done the picture making. Wanli Zhang is responsible for text editing. Jianing Hao did the work of device experimental design. Weizhou Chen is responsible for the environmental research.

\section{REFERENCES}

[1] Lin Fei, Analysis and Application of Humanized Design Concept in Industrial Design [J]. Art Science and Technology, 2017. (in

Chinese)

https://kns.cnki.net/kcms/detail/detail.aspx?db code $=$ CJFD \&dbname $=$ CJFDLAST2017 \&filen ame $=$ YSKK201707264\&v=r9qgtVC2CJsr8\% 25mmd2BgxsJgIAzH6F0NGdAUm3a07Dxdq VgvACq1dOdfBdmWwAajka5lx
[2] Wang Jiazhou, Research on the Design of Interactive Space in Urban Environment [D]. Nanjing Forestry University, 2013. (in Chinese) https://kns.cnki.net/kcms/detail/detail.aspx?db code $=$ CMFD $\&$ dbname $=$ CMFD201401 $\&$ filena me $=1013045906 . n h \& v=u 21 p f Q c z h c 1 d v I S 3 l e$ XcbF2VK05IqjFKSnV1AKIU40d56wuCn8vo wKs6zf2mOOow

[3] Chen Cong, Research on Tableware Experience Design [D]. Shanghai University, 2009. (in Chinese) https://www.docin.com/p177047533.html

[4] $\mathrm{Qu}$ Yukun, Research on the Application of Humanized Design in Product Design [J]. Industrial Design, 2020. https://kns.cnki.net/kcms/detail/detail.aspx?db code $=$ CJFD\&dbname $=$ CJFDLAST2020\&filen ame $=\mathrm{GYSH} 202002055 \& \mathrm{v}=\mathrm{rIHI} \% 25 \mathrm{mmd} 2 \mathrm{~B} 0$ qYfmdyMLC9gHuMcnybzmWjDi9LrRxp3N LO9hLXJ0N21Lvlt6CynecqxWIF

[5] Bai Junfeng, Wang Yaping, Zhang Tingting, Ergonomics Application in Design Practice Take a Toilet as an Example [J]. Industrial Design, 2018. (in Chinese) https://kns.cnki.net/kcms/detail/detail.aspx?db code $=$ CJFD $\&$ dbname $=$ CJFDLAST2018 $\&$ filen ame $=\mathrm{GYSH} 201805008 \& \mathrm{v}=4 \mathrm{KERGcbVjyZU}$ ZCvH8j\%25mmd2Bwd2kZU4FvZSwCu\%25 mmd2FU0oBMJtMmCAq9nU0TTN7sub\%25 mmd2FvfSIgR

[6] Fang Na, Li Keping, Humanized Design of College Dormitory Furniture [J]. Packaging Engineering, 2019. (in Chinese) https://kns.cnki.net/kcms/detail/detail.aspx?db code $=$ CJFD\&dbname $=$ CJFDLAST2019\&filen ame $=$ BZGC201910031 \&v=4W5apLBdGcmd AwWGbFB0aIYAawPuZy\%25mmd2BlF7tM $\%$ 25mmd2FFdpBJmrxDq2pHeZVrPfYcupxE VP 